\title{
The epidemiologic study of neonatal jaundice, relation between jaundice and liver and alternative methods to cure jaundice

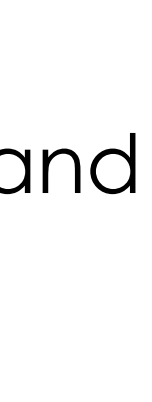

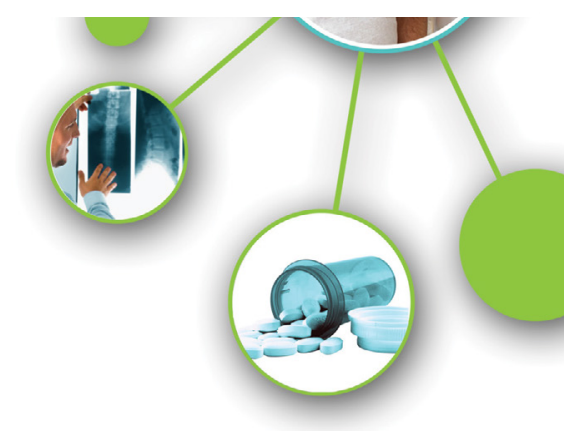

\begin{abstract}
Neonatal Jaundice is one of the most common diseases at birth. The goals of this study are understanding jaundice in newborns, the effects of this disease on the body; especially liver, and finally the treatment methods for this disease. According to the tests conducted since the discovery of the disease up to now, a significant percentage of new-borns at the very beginning or after several days of birth have a jaundice which, according to the conditions and factors influencing the disease, Bilirubin in newborn's blood, the severity of jaundice is different in each infant. The liver is the largest visceral structure in the abdomen and the largest gland in the body, with about 1500 grams weight in adults. Colour of the liver is reddish brown and it is located below the chest on the right side of the abdomen below the diaphragm. In the infant's body, new red blood cells are recovered every day. These red blood cells are broken down faster than the infant's liver can handle them. A yellowish substance called bilirubin, which is caused by the breakdown of red blood cells, is typically taken and removed from the blood by the liver. During pregnancy, the mother's liver receives bilirubin from the fetus's body and excretes it. But because the infant's liver did not grow sufficiently at the time of birth, it may be difficult to absorb and eliminate bilirubin from the infant's body, which is caused by jaundice in the new-born. In this article, we try to explain the liver, jaundice and its impact on newborns, and also treatment methods.
\end{abstract}

\section{Keywords: Neonatal jaundice, bilirubin, infant, liver, red blood cell, pregnancy, birth, disease}

\section{Introduction}

\section{- Jaundice and effects of light}

In the past and around the 1950s, blood transfusion was the primary treatment of hyperbilirubinemia or jaundice in infants. Hyperbilirubinemia or neonatal jaundice means the yellowish color of the skin in infants. Until the 1960s, phototherapy had not been used widely. At that time, blood transfusion was the only way to treat a new-born's jaundice. The effects of phototherapy and light on the treatment of neonatal jaundice were accidentally detected in the Essex hospital of England. A nurse working in the premature infant area realized that the infants admitted to her area, including fresh air and sunlight, had a lower level of bilirubin in their blood. Although this observation was the first time that they understood the effect of light on neonatal jaundice, it was taken seriously when a blood sample which was supposed to be sent to the laboratory for measuring bilirubin levels, accidentally left in a place next to the window and after that it was taken for testing. They found that the test result was not comparable with the jaundice of the infant and showed a lower level of bilirubin in the blood. In 1958, Cremer published his findings of the effect of lights on bilirubin of blood in the journal Lancet and introduced by Onishi in Japan in 1968. This theory was independently reviewed by the American medical group for about ten years after that $[1,2]$.

\section{- Neonatal jaundice definition}

Jaundice in infants is one of the most commonly occurring symptoms at birth, which means yellowish skin color in new-borns. It is a benign disease that can be treated at most after two weeks. All babies are born with an amount of bilirubin in their blood. About $65-75 \%$ of term neonates have jaundice, but in premature infants, this percentage increases and reaches to $80 \%$. Even the production of bilirubin is also present in the mother's womb, but it is transmitted through the mother's blood to the liver and is excreted by the liver. According to researches and studies, the percentage of

\section{Hamidreza Shirzadfar*, Kowsar Sheikhi and Zahra Meschian}

Department of Biomedical Engineering, Sheikhbahaee University, Isfahan, Iran

*Author for correspondence:

h.shirzadfar@shbu.ac.ir , h.shirzadfar@gmail.com 
jaundice in boys is higher than in girls. Jaundice occurs in babies three or four days after birth, which is when the baby is discharged from the hospital. For this reason, the doctor asks the infant's parents to examine the infant 4-5 days after birth. In some babies, only the white part of the eye is yellow, in others it is not only the eyes but also the skin is yellow. This material is collected and deposited in various tissues of the body, especially in the eyes and skin of the infant $[1,3,4]$.

\section{RBC decomposition and bilirubin formation}

In the baby's body, some red blood cells are recovered every day. These red blood cells are broken down faster than their liver can handle it and this process produces a yellowish substance called bilirubin. During pregnancy, the mother's liver receives bilirubin from the fetus's body and excretes it. But because the infant's liver did not grow sufficiently at the time of birth, it might have difficulties to absorb and eliminate bilirubin from the infant's body, which starts from a few days after birth and causes jaundice in babies. In addition, breast milk can also cause jaundice in the baby. Breastfeeding or some enzymes in breast milk will also cause jaundice [5].

FIGURE 1 shows levels of breaking red blood cells and the products of this decomposition. Hemoglobin is a protein that is in red blood cells and its' role is transporting oxygen and carbon dioxide in the blood. This protein has the haemo group, which is a prostatic group. In biochemistry, the prostatic group is a structure that attaches to proteins. For example, the haemo group in the hemoglobin structure is an example of a prostatic group. Hemoglobins have iron in their structure. Red blood cells are degenerated by splenetic macrophages after 120 days in the spleen. Hemoglobin in the red blood cells breaks down to haemo and globin after its destruction. The haemo loop also decomposes into a Biliverdin and a $\mathrm{CO}$ molecule. Biliverdin is a result of hemoglobin segmentation in red blood cells. The macrophages destroy red blood cells and break the bloodstream into Biliverdin. Biliverdin, produced by the Biliverdin reductase enzyme, is converted to Bilirubin [2,5] (FIGURE 2).

\section{Symptoms of jaundice in the neonates}

It is best for the baby's parents to take the baby's precise care in the first few days of birth [4]. The first part, which changes to yellow after jaundice, is the sclera in the baby's eyes, and then spreads to face, chest, and legs. The degree of the baby's jaundice can be approximated by color changes in each of these points. There are general symptoms of a neonate jaundice, such as yellow skin, yellow eyes (sclera), sleepiness, poor feeding, brown urine, fever, high-pitch cry, vomiting [1].

\section{Diseases caused by jaundice}

If the level of bilirubin goes high in blood and no treatment is given, it will cause problems for the infant. Some of these problems include $[6,7]$ :
1. Brain damage
2. Seizure
3. Cerebral palsy

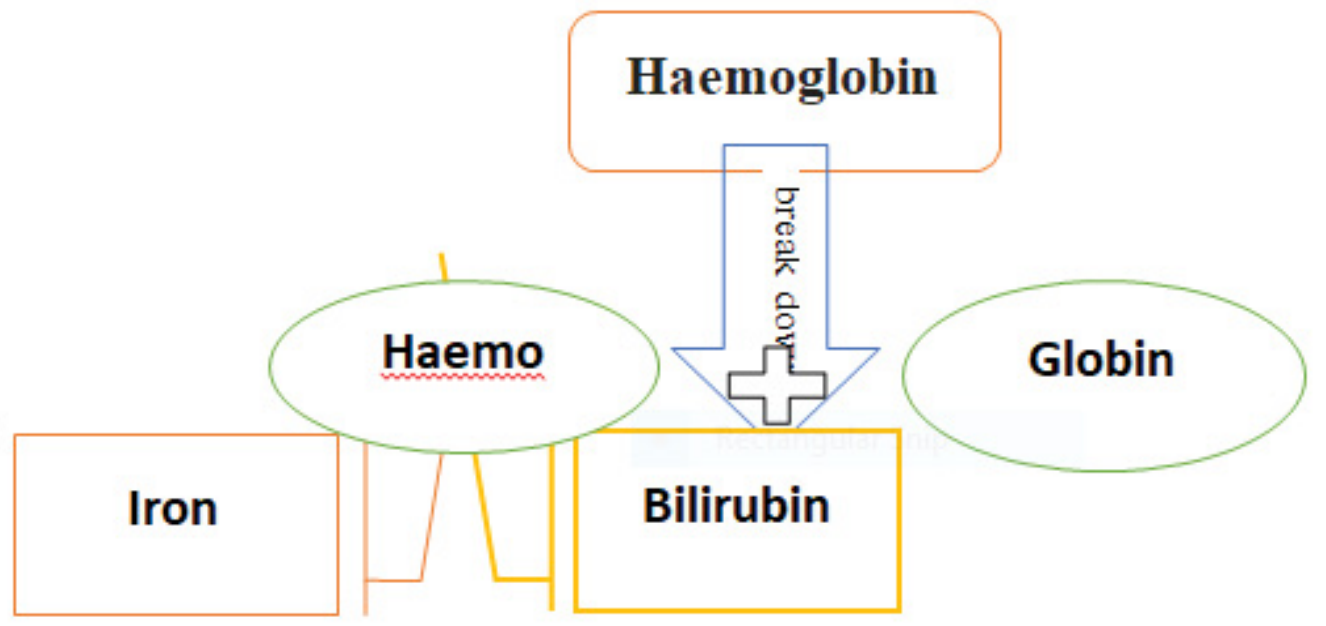

FIGURE 1. Breaking the red blood cell and producing bilirubin. 
FIGURE 2. Chemical structure of the haemo loop, the Biliverdin and Bilirubin molecule.

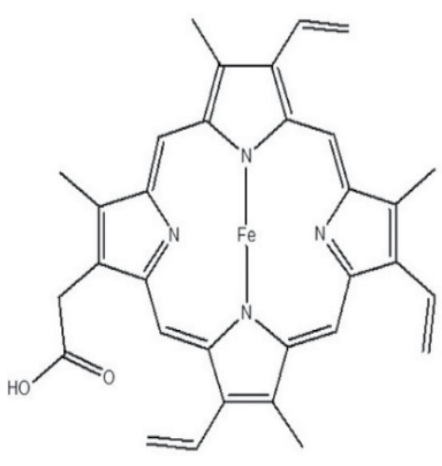

Heme oxygenase<smiles>C=CC1=C(C)/C(=C/c2[nH]c(/C=C3\N=C(/C=C4\NC(=O)C(C)=C4C=C)C(C)=C3CCC(=O)O)c(CCC(=O)O)c2C)NC1=O</smiles>

\section{Heme}

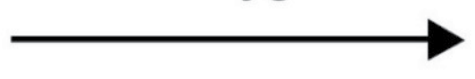

Biliverdin

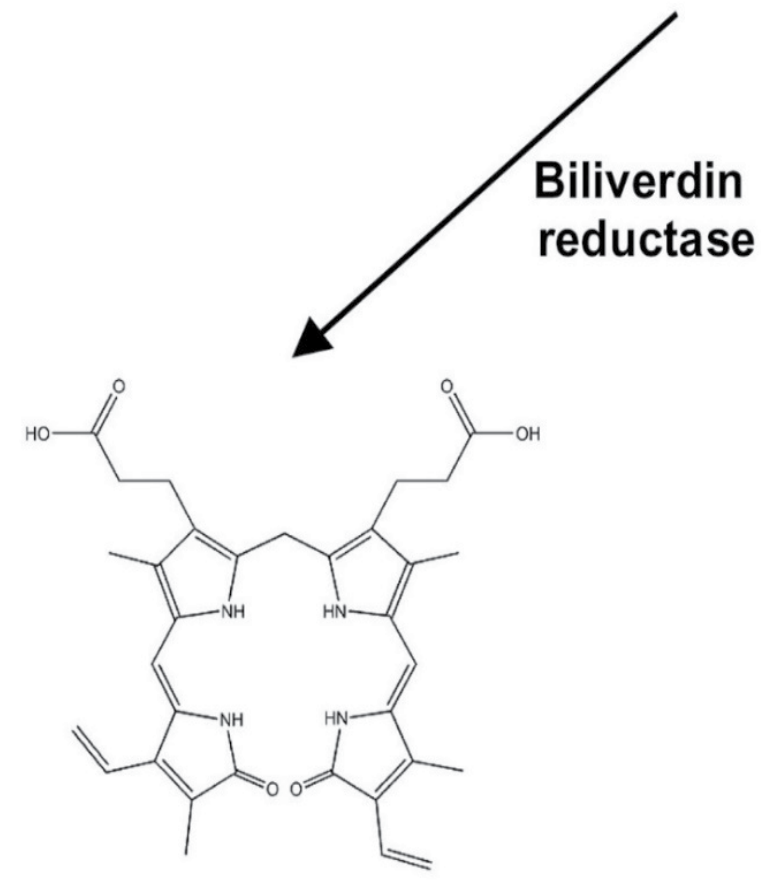

\section{Bilirubin}

4. Intellectual disability

5. Movement disorders

6. Dental anomalies

7. ADHD (Attention-Deficit Hyperactivity Disorder)

Types and Methods of Measuring Jaundice in Infants

\section{- Types of jaundice \\ Physiologic jaundice (natural)}

This type of jaundice is the most common type of jaundice in infants. This type of jaundice occurs because of the liver's disability in degradation of bilirubin. Generally, jaundice occurs in the early days of the birth of babies and disappears after 14 days or more [5].

\section{Jaundice in a premature infant}

This type of jaundice typically occurs in infants born sooner than the right time. The percentage of jaundice in preterm infants is higher than term infants [8].

\section{Neonatal jaundice from breast milk}

This type of jaundice occurs in a small percentage of infants. This is because of the material produced in the breast, which increases the level of bilirubin in the infant's body. These enzymes prevent the proper function of the 
baby's liver, which also prevents the effective elimination of bilirubin by the intestines of babies. This problem begins in the first days of baby's birth and milking [9].

Neonatal jaundice because of incompatibility of blood groups with the mother: The blood groups can be divided into two main ones, $\mathrm{ABO}$ and $\mathrm{RH} . \mathrm{ABO}$ system is divided into four main groups: $A, B, A B$ and $O$, and the $R H$ system is divided into two groups: positive $\mathrm{RH}$ and negative $\mathrm{RH}$. Your blood group consists of one of $\mathrm{ABO}$ and also one of $\mathrm{RH}$ groups. At the beginning of pregnancy, the mother's blood type is determined. For example, if the $\mathrm{RH}$ of the mother's blood is negative and the $\mathrm{RH}$ of the fetus is positive, the mother's body makes a substance against the blood of fetus. This substance in mother's blood can damage red blood cells of the fetus. If amount of this substance in mother's blood is low, infant may have brief jaundice after birth; But high amount of this substance can cause jaundice or severe swelling and anemia in infant that decreases total amount of red blood cells (RBCs) or hemoglobin in blood, or a lower ability of the blood to carry oxygen. This jaundice begins in the first days of the baby's life. Problems of $\mathrm{RH}$ can be resolved by infusion of $\mathrm{RH}$ immunoglobulin to the mother [9].

\section{- Methods of measuring}

A blood test is one of the methods that can determine the level of bilirubin in the blood. In recent years, doctors are using a device called Bili-Check for determining the level of bilirubin. This is a device for measuring the amount of jaundice and it is so easy to use. This device is used to detect bilirubin in the blood of infants through the skin and it does not require blood sampling from the infant. As a result, there is no pain or risk of infections for the infant, just by touching the baby's forehead lightly with the probe. This device directly measures the level of bilirubin in the baby's bloodstream and accurately displays the serum bilirubin levels of the blood after conversion to the digits [1,10-12].

\section{Liver}

The liver is the largest visceral structure in the abdomen and the largest gland in the body, with about 1500 gram weight in adults. The color is reddish brown, located below the chest on the right side of the abdomen below the diaphragm
(FIGURE 3). The liver has two large parts called the right and left edges. The gallbladder is located below the liver with pancreas and intestines. The liver and these organs work to synchronize, absorb and process food $[13,14]$.

As shown in FIGURE 4, two blood vessels are connected to the liver. First one is hepatic artery that provides $25 \%$ of liver blood and carries the aortic blood and the next one is a portal vein that provides $75 \%$ of liver blood and carries the blood, containing the digestive nutrients digested as well as the spleen and pancreas. These blood vessels are divided into capillaries and lead the blood to the lobules. Oxygen is supplied from both vessels. Almost half of the liver's oxygen is produced by portal veins and half by hepatic artery. The gallbladder is an organ located below the liver. Bile is a product secreted from the liver and stored in the gallbladder. During digestion, bile is released from the gallbladder and transported through the bile duct into the duodenum. For example, when a person eats a lot of fatty foods, like a steak, the body uses bile to decompose fat and digest it. Blood purification and bile production occur in the structural and functional parts of the liver [14,15-17].

As FIGURE 5 shows, portal veins and hepatic artery are mixed and enter the lobules then the blood penetrates into the liver lobules and is collected in the central vein or liver. The central veins are integrated and form the larger veins of the lobules, which finally connect to the liver vein. And the blood flows through the

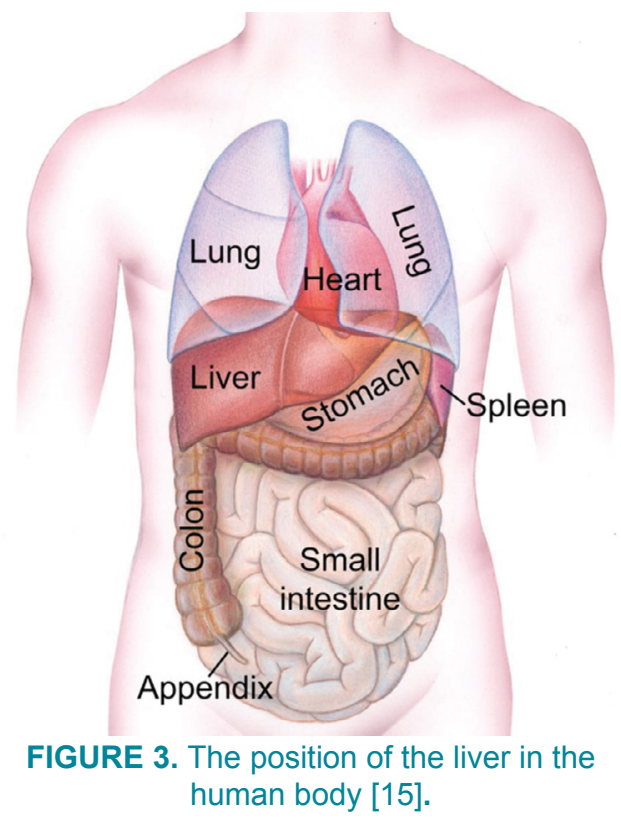


FIGURE 4. The internal anatomy of the liver [17].
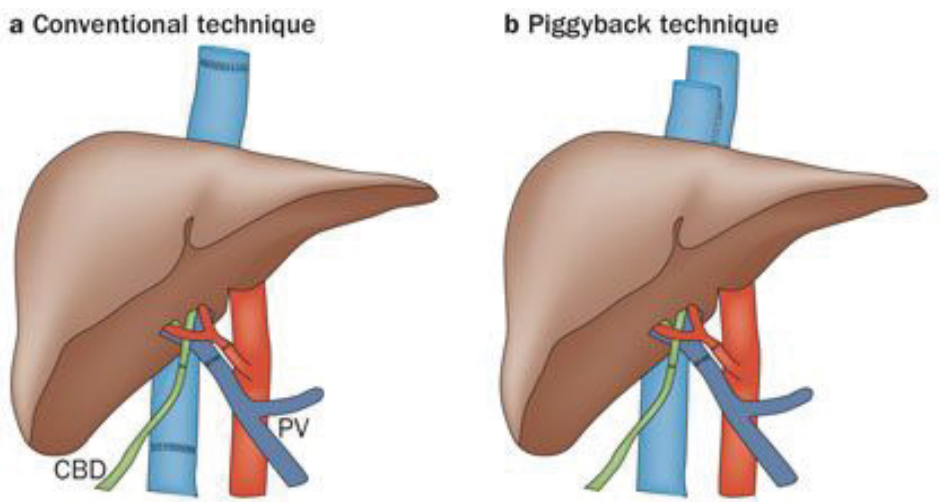

IVC inferior vena cava

HA hepatic artery

PV portal vein

CBD common bile duct

LHA left hepatic artery

LPV left portal vein

LHD left hepatic duct

LHV left hepatic vein

RHA right hepatic artery

RPV right portal vein

RHD right hepatic duct

RHV right hepatic vein

MHV middle hepatic vein

\section{c Split liver}

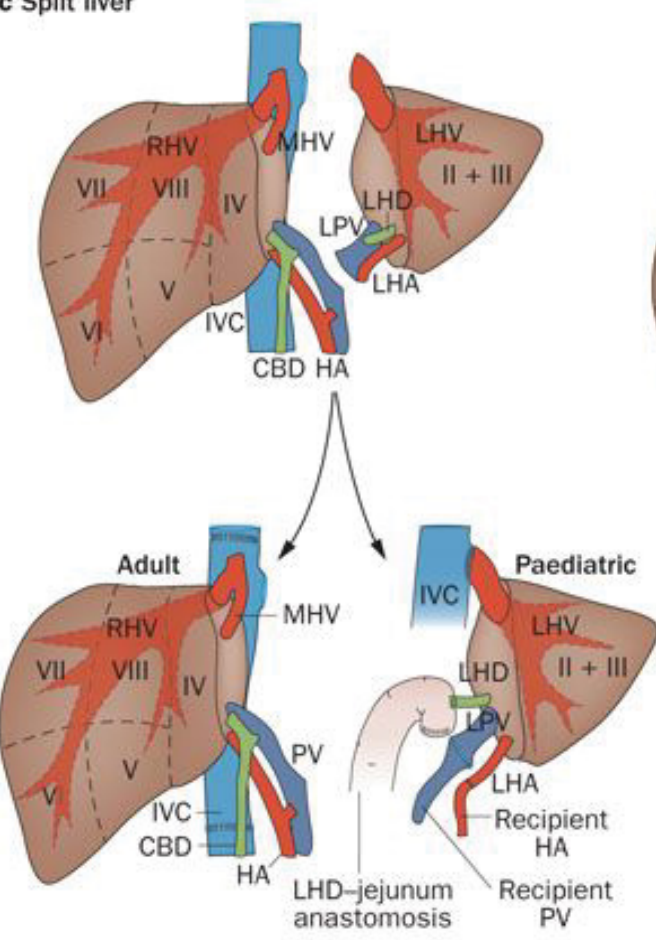

heart by inferior vena cava. If we cut a lobule of the liver in a transverse section, it is similar to a hexagon. The central vein is at the centre of the lobule, which collects blood from the lobule and transfers it to the hepatic artery. Around lobules, there are splints of the veins that supply the blood for it (portal vein and hepatic artery). There is also a bile duct here. Portal vein, hepatic artery and bile ducts finally form a three-port portal together $[14,18]$.

\section{- Liver tasks}

The main task of the liver is filtering the blood from the digestive system before it is transferred to the rest of the body. The blood from digestive organs goes to the liver through the portal vein. When the nutrients, drugs and toxic substances reach to the liver, they are processed, stored, d Living donor right lobe liver transplantation

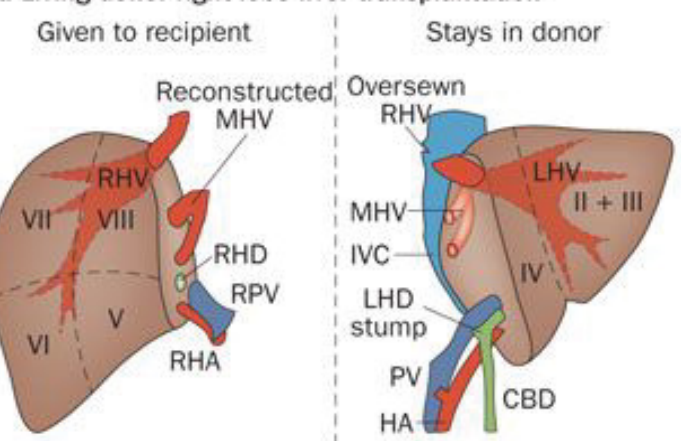

e Living donor left lobe liver transplantation

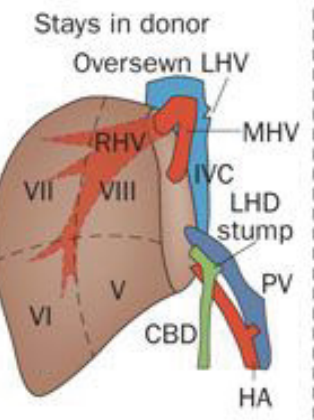

Given to recipient

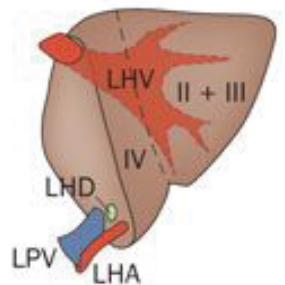

modified, detoxification and transmitted to blood or released in the intestine to disappear. The liver is very important to all metabolic processes in the body. In fat metabolism, liver cells decompose fats and produce energy. These liver cells also produce about $1000 \mathrm{ml}$ of bile per day. Bile plays an important role in decomposition and absorption of fats. In the metabolism of carbohydrates, the liver helps to keep blood glucose levels constant. For example, if blood glucose increases, the liver eliminates sugar from the alkali produced by portal veins and stores it in the form of glycogen. If the level of glucose is low, the liver breaks up the glycogen and transfers sugar to the blood. The liver also stores vitamins and minerals (such as iron, copper) and if it is necessary, transfers them to blood. The liver plays an important 


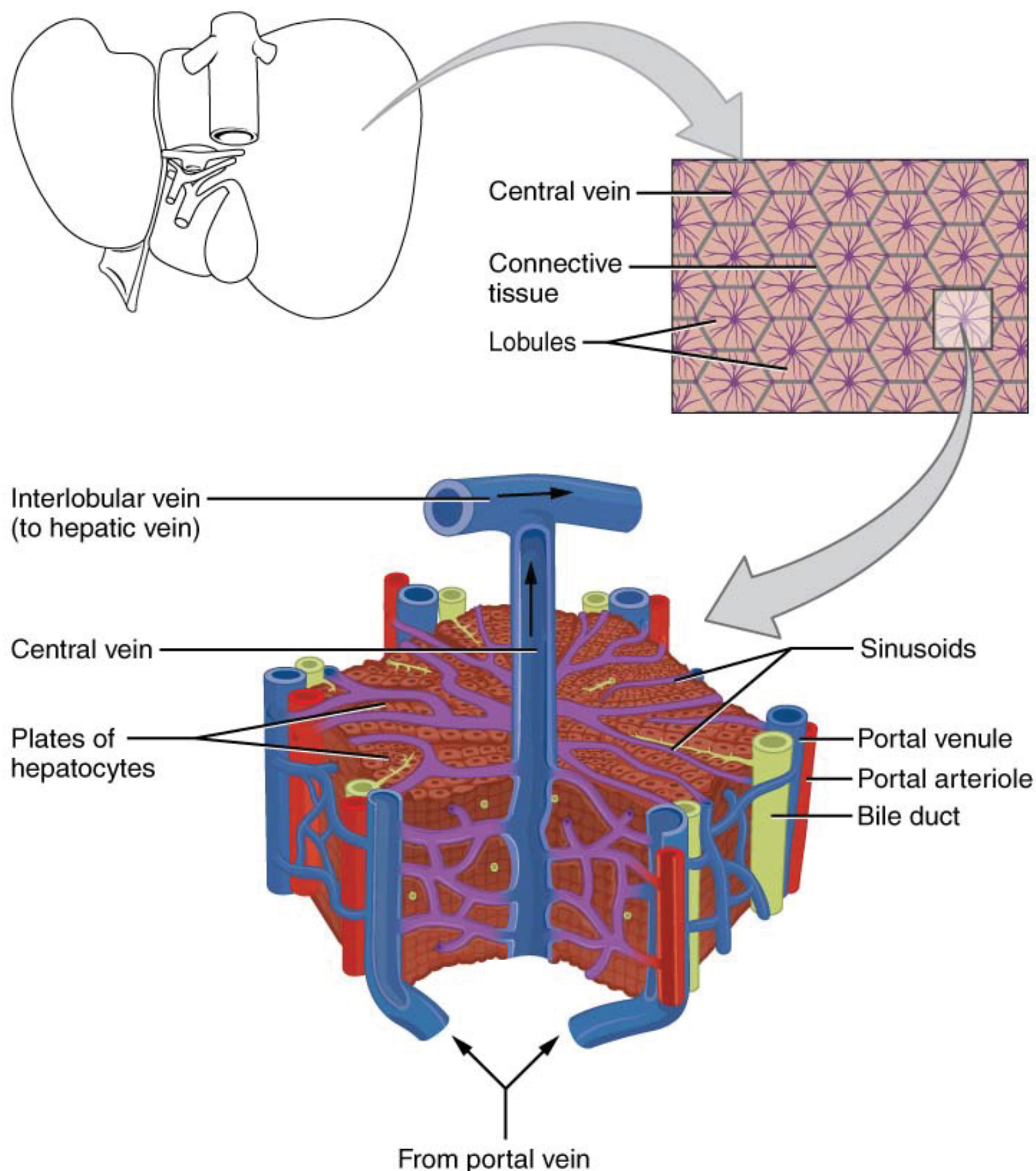

FIGURE 5. A liver lobule [18].

role in the metabolism of proteins too, so that the liver cells change amino acids in foods to produce energy, or produce carbohydrates or fats. A toxic substance called ammonia is a byproduct of this process. Liver cells convert ammonia into highly toxic substances that are called urea and is transmitted to the blood. Then urea is transferred to kidneys and it's removed by urine $[14,19]$.

\section{- Liver and jaundice}

As mentioned in the previous section, some new red blood cells are recovered in the baby's body every day. These red blood cells are broken down faster than infant's liver can handle them. A yellowish substance called bilirubin, which is caused by the breakdown of red blood cells, is typically taken and removed by the liver from the blood. During pregnancy, the mother's liver 
receives bilirubin from the fetus's body and excretes it. But because the infant's liver did not grow sufficiently at the time of birth, it may be difficult to absorb and eliminate bilirubin from the infant's body, which causes jaundice in the newborn [9].

\section{- Neonatal jaundice treatment Phototherapy device}

Jaundice will be dangerous for infants if the amount of bilirubin in their blood is 5 times more than their weight. In these conditions, phototherapies or, in more advanced conditions, blood transfusions should be performed [1]. One of the most effective and safe methods for treating neonatal jaundice is to use phototherapy. In these devices, they use fluorescent lamps, tungsten halogen bulbs, LEDs, optical fibers, etc. Bilirubin in our blood reacts to green and blue light. Greenlight has 495-570 nm wavelengths. Blue light has 495$450 \mathrm{~nm}$ wavelength and a limited absorption in the range of $450 \mathrm{~nm}$. Best lights for using in optical phototherapy devices are lights that have $400 \mathrm{~nm}$ to $520 \mathrm{~nm}$ wavelength so they usually use blue lights in phototherapy because it has limited absorption about 450 nanometers. In addition to the limited absorption of blue light, this color also has antimicrobial properties against bacteria $[1,20]$. It is difficult to determine the exact time of using phototherapy device for infants because every infant has special conditions and depends on amount and severity of jaundice in the infant blood and condition of every infant, such as:

\section{1) Age \\ 2) Level of bilirubin \\ 3) Premature or term infant}

Phototherapy should be continued until the level of bilirubin was decreased in infant's blood. Results of experiments on the amount of bilirubin in infant's blood and duration of phototherapy divide the new-borns into four groups: (These results were collected by taking 4 blue fluorescent tubes and $50 \mathrm{~cm}$ upper than infant's body. This is the standard way of phototherapy) (TABLE 1).

\section{Sunlight}

Unfortunately, phototherapy devices are not available all around the world. In some countries, because of climate or economic problems, there are not a lot of phototherapy devices. Direct sunlight can be effective in treating jaundice. Sunlight, in addition to its benefit, has a big problem too. It has large amounts of ultraviolet that can damage the skin of the infant. It also has many infrared rays which increase skin temperature and if there is not enough cooling equipment it can cause skin problems. To reduce these problems and improve treatment of jaundice in infants, filters have been made that placed upper than the infant's skin and preventing harmful rays from reaching the baby. At the same time, the beneficial rays in the treatment of jaundice pass through this filter. Sunlight filters are films that are made by plastic, The Air Blue 80 and Gila Titanium. Both films excluded virtually all UV-B and UV-A radiation. The Air Blue 80, chosen for use during overcast sky periods, and Gila Titanium film, chosen for periods of direct sunlight $[21,22]$.

\section{Blood transfusion}

Another way to treat jaundice in newborns is a blood transfusion, which should be done in a hospital by doctors. Blood transfusion is necessary in certain cases, when the infants are at risk for brain damage (brain cell deposition), or because phototherapy was not effective for them. Blood transfusion was developed during the 1940s by Louise Diamond and a group of surgeons at the Children's Medical Center in Boston, Massachusetts. As shown in FIGURE 6, in this method, they cut the umbilical vein and donated blood flows through the vein into the infant's body, and another tube is inserted to remove infant's blood slowly and replace with donor blood. These two tubes are connected to the vein by a valve. The amount of blood transmitted is usually more than the amount of blood in the infant's body, which is estimated $80 \mathrm{ml}$. This bilateral standard exchange eliminates about $85 \%$ of red cells and reduces bilirubin level to half. Blood transfusion lasts for at least 45 minutes. After two hours, bilirubin

\begin{tabular}{l|l}
13 to 15 & 16 to 17
\end{tabular}

\begin{tabular}{l|l}
18 to 20 & Above 20
\end{tabular}

\begin{tabular}{l|l}
17 to 18 & 23 to 24
\end{tabular}




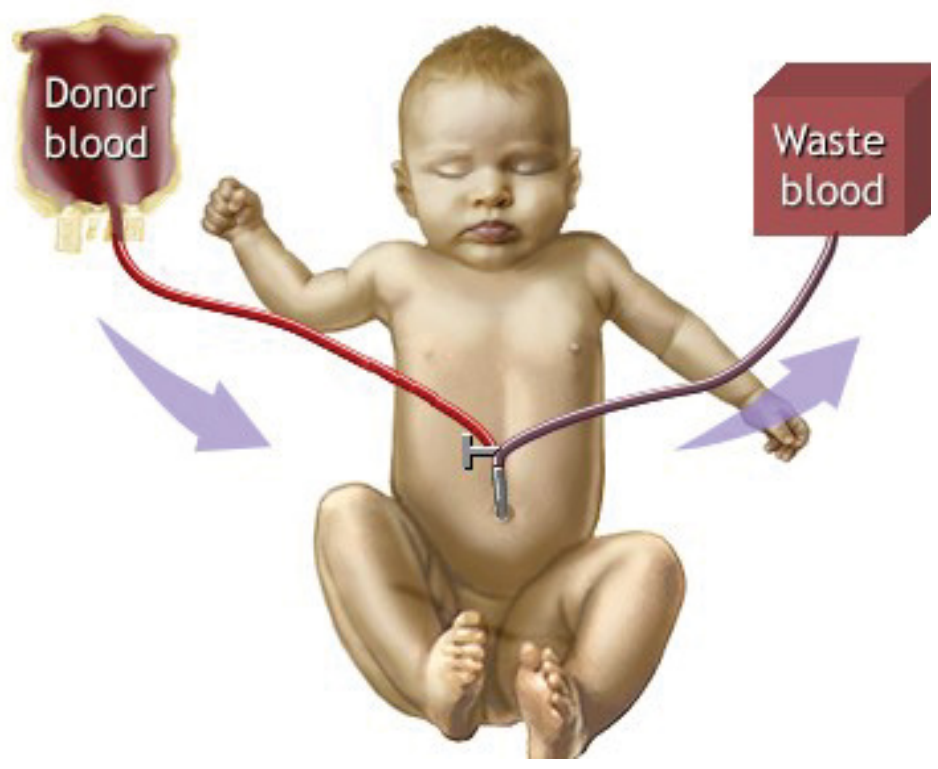

FIGURE 6: Exchange transfusion [28].

concentration in infant's blood reaches to half amount before the exchange. Therefore, the concentration of bilirubin in the blood should be examined 2 to 4 hours after exchanging, and then every 3 to 4 hours [23-26].

The amount of blood that the infant loses during each blood transfusion should be equal to the amount of blood that infuses the infant. This is done to prevent complications in the cardiovascular system, such as heart failure. This treatment should be repeated until the bilirubin decrease in the infant's blood. This method, like other therapies, also has probable side effects that may be caused by the exchange of blood in the body. Such as calcium deficiency, electrolyte imbalance, increasing potassium, infection and intestinal obstruction [25-28] (FIGURE 6).

\section{Conclusion}

Neonatal jaundice is one of the most common diseases at birth that threatens the health of the infant at the beginning days of birth. Studies show that jaundice in premature infants is more than term infants. It is about $80 \%$ in premature infants and in term infants, this percentage decreases to $60 \%$. As we said before, new red blood cells are recovered and broken down in the body every day. In the infant's body, the liver cannot eliminate these red blood cells and they produce yellowish substances that are called bilirubin. During pregnancy, the mother's liver receives bilirubin from the fetus's body and excretes it. But because the infant's liver did not grow sufficiently at the time of birth, it may be difficult to absorb and eliminate bilirubin from the infant's body, which causes jaundice in the new-borns. Neonatal jaundice has different types, such as; physiologic jaundice, jaundice in premature infants, the materials and enzymes in breast milk and incompatibility of blood groups ( $\mathrm{ABO}$ and $\mathrm{RH}$ ) between fetus and mother.

The first way to determine the level of bilirubin is the blood test. In recent years, the Bili-Check device is used instead of a blood test. Usually, phototherapy devices are used to decrease the level of bilirubin in infant's blood. In some countries, because of climate or economic problems, there are not a lot of phototherapy devices and they use sunlight. When the infants are at the risk of brain damage (brain cell deposition), or when phototherapy was not effective for them, blood transfusion is necessary. 


\section{References}

Yurdakök M. Phototherapy in the newborn: what's new? JPNIM. 4(2), 1-26 (2015).

Itoh $S$, Okada $\mathrm{H}$, Kuboi $\mathrm{T}$, et al. Phototherapy for neonatal hyperbilirubinemia. Pediatr. Int. 59(9), 959-966 (2017).

Maisels MJ, McDonagh AF. Phototherapy for neonatal jaundice. $N$. Engl. J. Med. 358(9), 920-928 (2008).

Maisels MJ, Newman TB. Jaundice in full-term and near-term babies who leave the hospital within 36 hours: the pediatrician's nemesis. Clin. Perinatol. 25(2), 295-302 (1998).

Dhumeaux D, Erlinger S. Hereditary conjugated hyperbilirubinemia: 37 years later. J. Hepatol. 58(2), 388-390 (2013).

Kapitulnik J. Bilirubin: an endogenous product of heme degradation with both cytotoxic and cytoprotective properties. Mol. Pharmacol. 66(4), 773-779 (2004).

Hashim ME. Evaluation of phototherapy with reflectors: A randomized controlled trial. Int. J. Pediatr. Adolesc. Med. 2(3-4), 117-122 (2015).

Hyperbilirubinemia. Management of hyperbilirubinemia in the newborn infant 35 or more weeks of gestation. Pediatrics. 114(1), 297 (2004).

Maisels MJ. Neonatal jaundice. Pediatrics in Review. 27(12), 443 (2006).

Jangaard K, Curtis H, Goldbloom R. Estimation of bilirubin using BiliChek ${ }^{\mathrm{TM}}$, a transcutaneous bilirubin measurement device: Effects of gestational age and use of phototherapy. Paediatr. Child. Health. 11(2), 79-83 (2006).

Poland RL, Hartenberger C, McHenry $\mathrm{H}$, et al. Comparison of skin sites for estimating serum total bilirubin in inpatients and out-patients: chest is superior to brow. J. Perinatol. 24(9), 541 (2004).

Ahmed M, Mostafa S, Fisher G, et al. Comparison between transcutaneous bilirubinometry and total serum bilirubin measurements in preterm infants <35 weeks gestation. Ann. Clin. Biochem. 47(1), 72-77 (2010).

Ellis H. Anatomy of the liver. Surgery (Oxford). 29(12), 589-592 (2011).

$\mathrm{Hu}$ J. Clinical anatomy of the liver, in Atlas of anatomic hepatic resection for hepatocellular carcinoma. Springer. 1-6 (2019).

Blum M, Feistel K, Thumberger T, et al. The evolution and conservation of left-right patterning mechanisms. Development. 141(8), 1603-1613 (2014).

Couinaud C. Liver anatomy: portal (and suprahepatic) or biliary segmentation. Dig. Surg. 16(6), 459-467 (1999).

Zarrinpar A, Busuttil RW. Liver transplantation: Past, present, and future. Nat. Rev. Gastroenterol. Hepatol. 10(7), 434-40 (2013).

https://teachmeanatomy.info/ abdomen/viscera/liver/

Li F, Tian Z. The liver works as a school to educate regulatory immune cells. Cell. Mol. Immunol. 10(4), 292 (2013).
Bhutani VK. Phototherapy to prevent severe neonatal hyperbilirubinemia in the newborn infant 35 or more weeks of gestation. Pediatrics. 134(3), 920 (2011).

Olusanya BO, Zainab OI, Cecilia $\mathrm{AM}$, et al. Maternal satisfaction with a novel filtered-sunlight phototherapy for newborn jaundice in Southwest Nigeria. BMC Pediatr. 14(1), 180 (2014).

Slusher TM, Olusanya BO, Hendrik J, et al. A randomized trial of phototherapy with filtered sunlight in African neonates. NEJM. 373(12), 1115-1124 (2015).

Alcock GS, Liley H. Immunoglobulin infusion for isoimmune hemolytic jaundice in neonates. Cochrane Database Syst. Rev. (2002).

Ahlfors CE. Criteria for exchange transfusion in jaundiced newborns. Pediatrics, 93(3), 488-494 (1994).

Patra K, Storfer IA, Siner B, et al. Adverse events associated with neonatal exchange transfusion in the 1990s. J. Pediatr. 144(5), 626-631 (2004).

Steiner LA, Bizzarro MJ, Ehrenkranz $\mathrm{RA}$, et al. A decline in the frequency of neonatal exchange transfusions and its effect on exchange-related morbidity and mortality. Pediatrics. 120(1), 27-32 (2007).

Jackson JC. Adverse events associated with exchange transfusion in healthy and ill newborns. Pediatrics. 99(5), e7 (1997).

http://www.floridahealthfinder. gov/healthencyclopedia/Health $\% 20$ Illustrated\%20Enc yclopedia/3/100018. aspx. 